\title{
CHARACTERIZATION OF SOME FULLY ORDERED RINGS*
}

\author{
M. SATYANARAYANA
}

(Received 27 May 1969)

Communicated by B. Mond

In a fully ordered (f.o.) ring with identity, the set of all bounded elements as defined below might be an Archimedian subring. Most of the examples of f.o. rings constructed in literature having the bounded set as Archimedian subring are polynomial rings. For example $I[x], R[x]$ etc., where $I$ is the ring of integers and $R$ is the field of rationals, with lexicographic ordering. Now we ask whether a f.o. ring with identity, with the set of bounded elements as Archimedian subring can be a polynomial ring over an Archimedian subring. This is answered affirmatively in Theorem 1. It is proved in Theorem 3 that f.o. rings with identity and with every positive element a large element, belong to the above class. The problem then arises as to when the set of all bounded elements, called a weak Archimedian subring in [2], becomes an Archimedian subring. This problem is completely solved in Theorem 2. The concept of weak Archimedian rings is found to be useful by the author in characterizing some f.o. rings as algebraic algebras in [3].

Notation. Throughout this paper all rings are assumed to be associative rings with identity. Convex ideals, lexicographic and full ordering are defined in the sense of Fuchs [1].

Definitions. An element $x$ in a f.o. ring $R$ with identity is said to be bounded if $|x|$ is less than some positive integral multiple of identity. Otherwise $x$ is called unbounded. $R$ is said to be weak Archimedian if every element of $R$ is bounded. Every f.o. ring with identity contains the maximal weak Archimedian subring. An element $x$ in a ring $R$ is said to be algebraic over a subring $S$ iff either $\sum_{i=0}^{n} a_{i} x^{i}$ $=0$ or $\sum_{i=0}^{n} x^{i} a_{i}=0$ for $a_{i} \in S$. If $x$ is not algebraic over $S$, then it is said to be transcendental over $S . R$ is $o$-simple if $R$ has no convex ideals.

THEOREM 1. Let $R$ be a f.o. ring with identity such that the maximal weak Archimedian subring $B$ (the set of all bounded elements in $R$ ) is an Archimedian subring. Then $R$ is either Archimedian or every element of $R-B$ is transcendental over $B$. Furthermore $R$ is an integral domain; $R$ is o-simple; if $B \neq R$, every element of $R-B$ is a non-unit and the Jacobson's radical $J(R)$ is a subset of $B$.

* Presented in the seminar on Algebra conducted by Delhi University in April, 1969. 
Proof. Let $x>0$ and $x \in R-B$. Suppose

$$
a_{n} x^{n}+a_{n-1} x^{n-1}+\cdots+a_{0}=0, \quad a_{i} \in B
$$

If $a_{n}<1$, since $B$ is Archimedian, there exists a positive integer $r$ such that $r a_{n}>1$. Then $r a_{n} x^{n}+\cdots=0$. Thus the leading coefficient can be taken $>1$. Let $a_{n}=$ $1+b, b>0$. There exists a $c>0, c \in B$ such that $c>1-a_{j}, j=0,1, \cdots n$. Since $x$ is unbounded $x>c$. So $a_{j}>1-c>1-x$. Hence

$$
0=a_{n} x^{n}+\cdots>(1+b) x^{n}+(1-x) x^{n-1}+\cdots>1+b x^{n}>1,
$$

which is a contradiction.

$R$ is an integral domain, since $x y=0 \Rightarrow x^{2}=0$ or $y^{2}=0 \Rightarrow x$ or $y \in B$ by the above result. However, no element of $B$ is nilpotent.

Let $A$ be a non zero convex ideal in $R$. If $A \neq R$, then $A \cap B=0$, since, otherwise $A \cap B \neq 0 \Rightarrow A \cap B$ is a non zero convex ideal in the Archimedian ring $B$. Hence $A \cap B=B$ and thus $B \subset A, 1 \in A$ and $A=R$. Now, if $x>0$, and $x \in A, x \notin B$ from the above. Hence $x>1$, which implies $1 \in A$, since $A$ is convex. Thus $A=R$ and $R$ is 0 -simple. Let $x>o ; x \in R-B$ and $x$ be a unit. Evidently $x>1$. Then $1=x x^{-1} \geqq x^{-1} \Rightarrow x^{-1} \in B$. Then $x$ is algebraic over $B$, a contradiction.

To prove $J(R) \subseteq B: x>0, x \in J(R) \Rightarrow(1+x)$ is a unit $\Rightarrow 1+x \in B$ from the above $\Rightarrow x \in B$.

Definition. An element $x$ in a f.o. ring with identity is said to be large, if, for any natural number $m$, there exists a natural number $N$ such that $N|x|>m$.

REMARK 1. If every positive element is large in a f.o. ring with identity, then the ring need not be Archimedian nor even weak Archimedian. For example let $I[x]$ be a polynomial ring over the ring of integers, fully ordered by setting $a_{0}+a_{1} x+\cdots+a_{n} x^{n}>0$ iff $a_{n}>o$ or $a_{n}=o, a_{n-1}>0 \cdots$ etc. Every positive element $\sum a_{n} x^{n}, a_{n} \neq o$, is greater than every natural number and hence is large. Also by the same reason, this ring is not weak Archimedian and hence not Archimedian.

REMARK 2. If a f.o. ring with identity is weak Archimedian, then every positive element need not be large. Consider $I[x]$ as above. Set $a_{0}+a_{1} x+\cdots+a_{n} x^{n}>o$ iff $a_{0}>0$ or $a_{0}=o, a_{1}>o \cdots$ etc. This ring is weak Archimedian. Since $N x<1$ for every natural number $N, x$ is not large.

Now in the following theorem, we obtain the necessary and sufficient conditions for a weak Archimedian ring to become an Archimedian ring. It can easily be verified that Archimedian rings are $o$-simple and every positive element is large.

THEOREM 2. Let $R$ be a weak Archimedian ring with identity. Then $R$ is Archimedian if either one of the following conditions is satisfied: 
i) every positive non-unit is large,

ii) $R$ is o-simple.

Proof. By virtue of the foot-note $[1 ;$ p. 12] it suffices to prove that, for every $a>o, b>o$ there exists a natural number $N$ such that $N a>b$, in order to establish that $R$ is Archimedian.

Assume (i). Let $a>o, b>o$. Let $a<b$. Suppose that $a$ is a non-unit. By weak Archimedian property, $b-a<m, m$ being a natural number. Then $b<a+m \Rightarrow b<a+N a$, where $m<N a$ since $a$ is large. Thus $(N+1) a>b$.

If $a$ is a unit, $a<b \Rightarrow 1<b a^{-1} \Rightarrow b a^{-1}-1>o \Rightarrow b a^{-1}-1<m, m$ being a natural number, by the weak Archimedian property $\Rightarrow b<(m+1) a$. Thus (i) implies that $R$ is Archimedian.

Assume (ii). By virtue of (i) it suffices to show that every positive non-unit is large. Assume the contrary, that there exists a positive non-unit $x$ and a natural number $m$ such that $N x<m$ for every natural number $N$. If $A=\{y|| y \mid \leqq$ some element in $R x\}$ then $A$ is a non-zero left ideal since $A \supset R x$ and is convex. Since $R$ is $o$-simple, $R$ has no proper convex left ideals $[1 ; \mathrm{p} .132$, Theorem 9]. So $A=R$. Then $1 \leqq t x, t>0$ and $m \leqq m t x \leqq r x$ since $m t<r, r$ being a natural number, by the weak Archimedian property. This is a contradiction since $m>N x$ for every natural number $N$.

Now condition (i) of Theorem 2 yields the following result.

THEOREM 3. If every positive element is large in a f.o. ring $R$ with identity, then the maximal weak Archimedian subring of $R$ is Archimedian and its characterization is determined by Theorem 1.

REMARK 3. We have proved in Theorem 1, if $S$ is the maximal weak Archimedian subring of a f.o. ring $R$ with identity and if $S$ is Archimedian, then $R$ is $o$-simple. It seems probable that the converse, namely, if $R$ is $o$-simple, then $S$ is Archimedian, might be true. But the author is unable to prove this. However to obtain this, it suffices to show that $S$ is $o$-simple if $R$ is $o$-simple by virtue of Theorem 2.

\section{References}

[1] L. Fuchs, Partially ordered Algebraic systems, Pergamon Press, Addison -Wesley (1963).

[2] M. Satyanarayana, 'Weak Archimedian Rings', Mathematische Nachrichten. 41 (1960), 133-137.

[3] M. Satyanarayana, 'Fully ordered Rings', Mathematische Nachrichten. 44 (1970), 199-204.

S.V. University, Tirupati, India

Bowling Green State University, U.S.A. 